\title{
Fetal Hydrops in Combination with Gonadoblastoid Testicular Dysplasia May Represent a Lethal Type of Noonan Syndrome
}

Theresa Reischer ${ }^{1}$, Maximilian Schmid ${ }^{1}$, Sukirthini Balendran ${ }^{1}$, Manuel Nistal$^{2}$, Julia Vodopiutz ${ }^{3}$, Elisabeth Krampl-Bettelheim ${ }^{4}$, Niko Popitsch $^{5}$, Sandra Liebmann-Reindl ${ }^{6}$ and Berthold Streubel ${ }^{6,7 *}$

${ }^{1}$ Department of Obstetrics and Feto-Maternal Medicine, Medical University of Vienna, Vienna, Austria

${ }^{2}$ Department of Pathology, Hospital La Paz, Universidad Autónoma de Madrid, Spain

${ }^{3}$ Department of Pediatrics, Medical University of Vienna, Vienna, Austria

${ }^{4}$ Fetomed - Center for Fetal Medicine, Doebling Private Hospital, Vienna, Austria

${ }^{5}$ Wellcome Trust Centre for Human Genetics, University of Oxford, Oxford, UK

${ }^{6}$ Core Facility Genomics, Medical University of Vienna, Vienna, Austria

${ }^{7}$ Department of Pathology, Medical University of Vienna, Austria

\begin{abstract}
Objectives: Fetal gonadoblastoid testicular dysplasia is a rare finding. The combination with typical features of Noonan syndrome has never been described so far. We performed genetic testing including whole exome sequencing in two cases with fetal hydrops, congenital heart disease and gonadoblastoid testicular dysplasia.

Methods: Exome sequencing was performed in the index case, where high quality DNA was isolated from fetal blood. In the second case and in five further gonadoblastoma samples, conventional Sanger sequencing was performed on DNA isolated from formalin fixed, paraffin embedded tissue.

Results: Whole exome sequencing of the index case revealed a pathogenic mutation in the RIT1 gene (c.270G >A (p.Met90lle)), leading to the diagnosis of Noonan syndrome type 8 . In case 2, Sanger sequencing of RIT1 did not show any disease causing mutations. Sequencing of PTPN11 revealed a heterozygous frameshift mutation (c.1098delA, p.K366Nfs*12). Successful sequencing of four gonadoblastoma samples revealed mutations in RIT1 in two cases.

Conclusion: Here we present a lethal form of Noonan syndrome in two fetuses with typical features combined with severe hydrops and gonadoblastoid testicular dysplasia. Gonadoblastoid testicular dysplasia may be an additional feature of Noonan syndrome, which has not been described so far, but analysis of more cases is needed. Moreover, we have demonstrated a potential role of RIT1 in the pathogenesis of gonadoblastoma.
\end{abstract}

Keywords: Fetal gonadoblastoid testicular dysplasia; Fetal hydrops; Noonan syndrome; PTPN11; RIT1

\section{Introduction}

Noonan syndrome is a heterogeneous genetic disorder characterized by craniofacial features, heart defects and short stature. Furthermore, affected individuals often present skeletal defects, delayed psychomotor development, intellectual impairment, increased bleeding tendency and cryptorchidism. Noonan syndrome is inherited in an autosomal dominant manner, whereas in around $60 \%$ of cases the mutations arise de novo. At present, genetic diagnosis can be made in approximately $70 \%$ of suspected cases. Germline mutations in genes of proteins involved in the RAS/mitogen-activated protein kinase (MAPK) signaling pathway have been described in Noonan syndrome [1]. This pathway is essential for the regulation of cell proliferation, differentiation and survival [2]. Approximately $50 \%$ of the known mutations are located in the PTPN11 gene. Other genes known to be involved in the pathogenesis of Noonan syndrome include RAF1, SOS1, KRAS, BRAF, NRAS, MAP2K and SHOC [1,2]. Recent investigations suggest that mutations in the RIT1 gene might also be causal for Noonan syndrome [3].

Prenatally, features of Noonan syndrome may include increased nuchal translucency, cystic hygroma, polyhydramnios, congenital heart disease, renal abnormalities and occasional abnormal maternal serum triple screen [4]. Few cases of Noonan syndrome have been reported with signs of fetal hydrops and severe perinatal outcome. These cases were associated with specific mutations in the PTPN11, RAF1 and SHOC2 genes, which have all been described as somatic mutations in cancer as well [5-7]. Here we describe two cases of fetal lethal Noonan syndrome presenting with hydrops and gonadoblastoid testicular dysplasia (FGTD). Fetal gonadoblastoid testicular dysplasia is a very rare condition, so far described in seven cases [8-10]. This lesion bears a strong phenotypic resemblance to gonadoblastoma, a type of germ cell tumor typically found in streak gonads of patients with existent gonadal dysgenesis [11]. In contrast, FGTD is not associated with gonadal dysgenesis or abnormal gonadal determination. Previously, the only defined disorder associated with FGTD was Walker-WarburgSyndrome [9]. FGTD has recurrently been described in fetuses with severe hydrops and normal karyotype. We performed whole exome sequencing in a fetal case of FGTD and identified the disease causing mutation in RIT1, which had already been described in Noonan syndrome previously. Targeted resequencing of a second case featuring FGTD identified a mutation in PTPN11, the gene most frequently mutated in Noonan syndrome. Additionally, we tested DNA extracted

*Corresponding author: Berthold Streubel, Department of Pathology, Medical University of Vienna, Waehringer Guertel 18-20 1090 Vienna, Austria, Tel: 0043-1 4040078340; E-mail: berthold.streubel@meduniwien.ac.at

Received August 01, 2016; Accepted September 21, 2016; Published September 23, 2016

Citation: Reischer T, Schmid M, Balendran S, Nistal M, Vodopiutz E, et al. (2016) Fetal Hydrops in Combination with Gonadoblastoid Testicular Dysplasia May Represent a Lethal Type of Noonan Syndrome. Human Genet Embryol 6: 137. doi:10.4172/2161-0436.1000137

Copyright: (c) 2016 Reischer T, et al. This is an open-access article distributed under the terms of the Creative Commons Attribution License, which permits unrestricted use, distribution, and reproduction in any medium, provided the original author and source are credited. 
from formalin fixed, paraffin embedded (FFPE) gonadoblastoma tissue and detected somatic RIT1 mutations.

\section{Materials and Methods}

\section{Clinical cases}

Our study consists of the investigation of two fetal cases, the index case (case 1) and a second case with FGTD (case 2), which was published and described by Nistal et al. previously [8]. Case 1 presented at 13 weeks of gestation for first trimester combined screening. The ultrasound revealed an increased nuchal translucency (NT) of $10.2 \mathrm{~mm}$ (Figure 1a), and a right aortic arch was suspected. During the second trimester, further ultrasound examinations including fetal echocardiography showed a pronounced cystic hygroma, a polyhydramnios and evidence of a right aortic arch without other associated cardiac malformation. Additional fetal magnetic resonance imaging at 22 weeks of gestation confirmed the previous findings and detected marginally enlarged posterior lateral cerebral ventricles. After detailed counseling, the couple decided to continue the pregnancy despite these abnormalities. At 27 weeks of gestation, the woman presented with vaginal bleeding and contractions. Due to persistent contractions and laboratory evidence of inflammation, caesarian section was performed. The baby was transferred to the neonatal intensive care unit where it passed away on the second day of life as a result of respiratory failure. Post mortem examination showed a pronounced fetal hydrops with massive pleural and pericardial effusion, lung hypoplasia, interstitial pulmonary emphysema and multifocal lung hemorrhages. The heart featured a double aortic arch (hypoplastic left arch) with retro esophageal ring formation and a bicuspid aortic valve. Facial dysmorphic features included down slanting palpebral fissures and small low-set, posteriorly rotated ears. Histological examinations showed hemorrhage in various organs including the gastrointestinal tract and the central nervous system, thus implying a coagulant dysfunction. Furthermore, gonadoblastoid dysplasia was described in one of the testes. Routine prenatal cytogenetic analysis with Giemsa banding revealed a normal male karyotype. Subsequent chromosomal microarray analysis detected no evidence of a clinically relevant microdeletion or microduplication and excluded uniparental disomy. Gene analysis of PTPN11, RAF1 and KRAS was performed for suspected Noonan syndrome, but no pathogenic variants were found in these genes. High quality DNA isolated from fetal blood was available for whole exome sequencing (WES).

Case 2 was previously described by Nistal et al. [8]. A 33 year old woman gave birth to a male fetus at $26+6$ weeks of gestation. Prenatally, a polyhydramnios was present and chromosomal analysis revealed a normal male karyotype. The post mortem examination showed a generalized edema and evidence of fetal hydrops. Craniofacial dysmorphism was described including a flattened nose, low-set ears and stenosis of the external ear canal. Additionally, lung hypoplasia and a ventricular septal defect were present. The left kidney presented with cystic dilatation and histological examination confirmed the diagnosis of hydronephrosis. Furthermore, the brain featured mild ventricular dilatation and focal hemorrhages in the basal nuclei. FGTD was described in detail in the publication of Nistal et al. We extracted DNA from stored fetal FFPE tissue, provided by the group of Nistal [8]

In addition to two fetal cases of Noonan syndrome with FGTD, we tested another five cases of gonadoblastoma (G1-G5) for somatic mutations in the RIT1 and the PTPN11 gene. The main patient characteristics and their precise diagnoses are shown in Table 1.

\section{Exome sequencing}

Exome sequencing was performed in the index case where high quality DNA was isolated from fetal blood. Exome enrichment was carried out using the SureSelectXT All Exon, V5 kit (Agilent). Sequencing was performed on a HiSeq 2000. High-throughput sequencing resulted in 60 Mio $100 \mathrm{bp}$ paired-end reads that were quality-checked and mapped to the human hg19 reference genome.
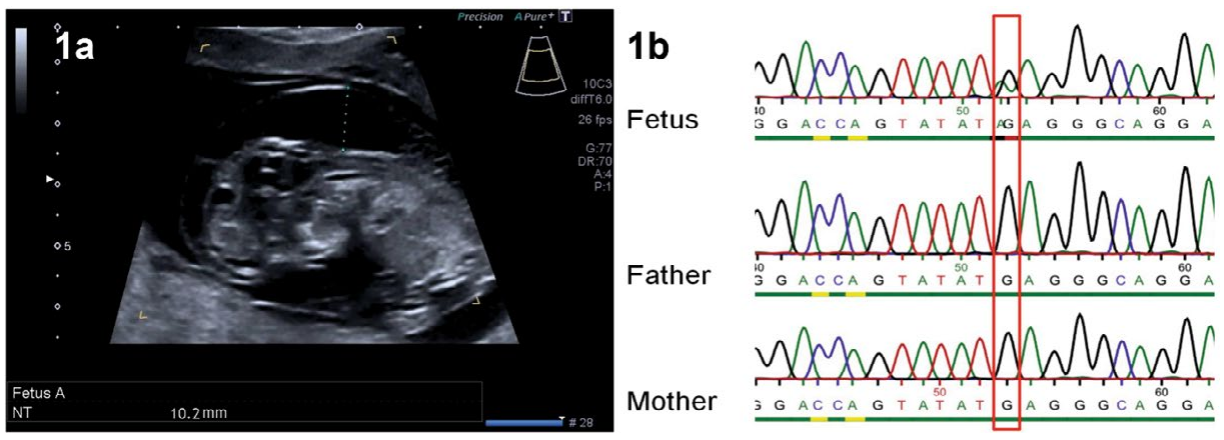

Figure 1: Index Case 1a: Ultrasonography at 13 weeks of gestation, showing an increased nuchal translucency (NT) of 10.2 mm. $1 \mathrm{~b}$ : Sanger sequencing confirms a heterozygous c.270G>A RIT1 mutation in the fetus. Parents show the wild type (G) at this position (red rectangle).

\begin{tabular}{|c|c|c|c|c|c|c|}
\hline \multicolumn{3}{|c|}{ Clinical Data } & \multirow{2}{*}{ Diagnosis } & \multirow{2}{*}{$\begin{array}{l}\text { Biopsy } \\
\text { Material }\end{array}$} & \multicolumn{2}{|c|}{ Sequencing Data } \\
\hline Case & Sex & Age (Y) & & & \multirow{2}{*}{$\begin{array}{l}\text { RIT1 Analysis } \\
\text { Analysis Failed }\end{array}$} & \multirow{2}{*}{$\begin{array}{c}\text { PTPN11 Analysis } \\
\text { Analysis Failed }\end{array}$} \\
\hline G1 & & & No Data Available & & & \\
\hline G2 & Male & 1 & Gonadal Dysgenesis and Gonadoblastoma & Testicle & Negative & Negative \\
\hline G3 & Female & 18 & $\begin{array}{l}\text { Gonadal Dysgenesis of the Right Ovary Gonadoblastoma of The Left } \\
\text { Ovary }\end{array}$ & Left Ovary & $\begin{array}{l}\text { C. }-43-1 G>A \\
\text { C-85G }>A(P . A 29 T)\end{array}$ & Negative \\
\hline G4 & Female & 12 & Dysgerminoma and Gonadoblastoma & Right Ovary & $\begin{array}{l}\text { C.473G >A, P.C158Y } \\
\text { (Homozygous) }\end{array}$ & Negative \\
\hline G5 & Female & 18 & Gonadal Dysgenesis Dysgerminoma, Gonadoblastoma & Ovar/Testicle & Negative & Negative \\
\hline
\end{tabular}

Table 1: Clinical data and sequencing results of gonadoblastoma samples (G1-G5). 


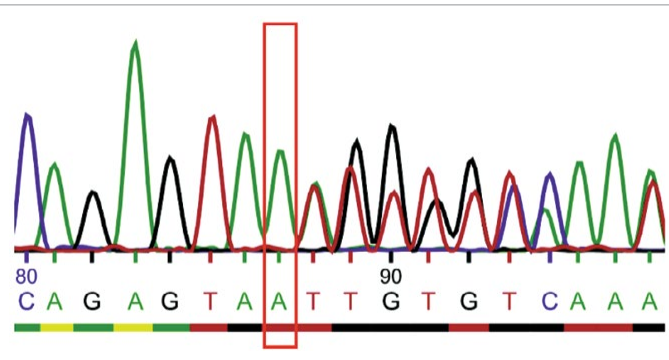

Figure 2: Case 2: Sanger sequencing of PTPN11 identified a heterozygous frameshift mutation in exon $10 \mathrm{c} .1098 \mathrm{delA}\left(\mathrm{p} . \mathrm{K} 366 \mathrm{Nfs}{ }^{* 12}\right)$ causing a premature stop codon.

We determined single nucleotide variations (SNV) and insertions and deletions (INDEL) from the data by combining the results of multiple read mapping and variant-calling software: First, reads were aligned using three different short-read mappers (bwa [12], bowtie2 [13] and NextGenMap [14], which aligned between $93-96 \%$ of the read pairs. Reads with low mapping quality $(\mathrm{MAPQ}<30)$ were removed, INDEL realignment was performed using the Genome Analysis Toolkit (GATK) [15] and PCR duplicates were removed using picard tool [16]. Per-base quality scores were recalibrated using GATK and the resulting alignments were quality-checked. These final alignments contained 56-58 Mio mapped paired-end reads. Two different variantcalling tools (samtools [17] and GATK UnifiedGenotyper) were applied to the alignments, which resulted in a total of six mapper/ variant-caller combinations per data set. These sets were then merged by including only single nucleotide polymorphisms (SNP) that were identified by at least $50 \%$ of these combinations. Low-quality GATK variant calls were filtered out. The variant sets were comprehensively annotated using annovar (Version 21.02.21013 [18]) and CODOC [19] and stringently filtered: First, only exonic (also ncRNA) variants and variants near known splice sites were considered and synonymous SNVs were excluded. All remaining variants that were considered "benign" by at least two of the three in silico SNP effect prediction tools applied (SIFT, MutationTaster and PolyPhen-2) were removed. This set was then further filtered by excluding all variants in low-coverage regions (coverage at the variant position at least five for homozygous variants and at least 13 for heterozygous calls). Candidate variants were confirmed by conventional Sanger sequencing.

\section{RIT1 and PTPN11 analysis}

RIT1 and PTPN11 sequencing was performed in case 2 and in five gonadoblastoma samples (G1-G5), where only FFPE material was available. DNA was isolated from FFPE spleen tissue (case 2) and FFPE gonadal tissue (G1-G5) using QIAamp DNA FFPE Tissue kit (Qiagen). DNA quantification was performed with the Qubit 2.0 fluorometer. Conventional Sanger sequencing of RIT1 and PTNPN11 was implemented in line with published data [20]. Primer sequences and details on PCR conditions can be provided on request.

\section{Results}

\section{Exome sequencing of case 1 identifies a disease causing mutation in RIT1}

In case 1, variant-calling tools identified 181749 variants, of which 23697 (13\%) were found in exonic regions. A total of 7074 out of the 23697 variants remained after the exclusion of synonymous and benign variants. Out of these remaining 7074 variants 6898 were previously reported to dbSNP (b138) without pathogenic relevance for the clinical phenotype. The remaining 176 variants with potential pathogenic effect were correlated with known Mendelian disorders and type of inheritance, gene function, gene expression pattern, and in silico prediction tools as outlined above. The heterozygous missense mutation NM_006912.5: c.270G>A (NP_008843.1: p.Met90Ile) was the only remaining pathogenic variant after WES and the described analyses. Sanger sequencing of the RIT1 gene was performed of the fetal DNA and both parents. Sanger sequencing confirmed the heterozygous mutation in the fetus. Both healthy parents were negative for the mutation (Figure 1b). Thus, we conclude that the fetus acquired a de novo mutation for autosomal dominant Noonan syndrome type 8 (OMIM \#615355).

\section{Sanger sequencing reveals a pathogenic PTPN11 mutation in case 2}

Based on our findings in case 1 and due to the large phenotypic overlap of cases 1 and 2, we first tested for RIT1 mutation in case 2. Sanger sequencing of RIT1 did not show any disease causing mutation. Due to the poor DNA quality WES could not be applied in this case, wherefore we performed Sanger sequencing for PTPN11, the gene most frequently mutated in Noonan syndrome. We identified a heterozygous frameshift mutation in exon 10 NM_002834: c.1098delA (p.K366Nfs`12) causing a premature stop codon (Figure 2).

\section{RIT1 mutations in gonadoblastoma}

Sequencing analysis of RIT1 in gonadoblastoma samples was successful in four out of five cases (G1-G5). Case G3 showed two heterozygous mutations, more precisely a missense mutation: c. $85 \mathrm{G}>\mathrm{A}$ (p.A29T) and a frameshift mutation leading to a premature stop codon: c.43delC. Case G4 revealed a homozygous missense mutation: c.473G>A, (p.C158Y). G2 and G5 did not show any mutation in RIT1 and in G1 sequencing analysis failed due to poor DNA quality. Sanger sequencing of PTPN11 in these samples did not show any pathogenic variant. In summary, successful sequencing of four gonadoblastoma samples revealed RIT1 mutations in two cases (Table 1).

\section{Discussion}

In our index case, we describe the first case of a fetus with RIT1 mutation leading to fetal hydrops and lethal outcome. The first RIT1 mutation in Noonan syndrome was discovered by Aoki et al. [3] by means of WES of postnatal cases featuring the typical symptoms. Subsequent mutations were identified from larger cohorts, resulting in a detection rate of $9 \%$ in individuals diagnosed with Noonan syndrome without detectable mutations in known disease related genes [3]. Here, we describe the first prenatal case of Noonan syndrome associated with a RIT1 mutation. The p.Met90Ile RIT1 variant of our index case was described in a 3 year old girl by Aoki et al. and in a 2 year old boy by Koenighofer et al. with the two cases presenting distinct phenotypes. Both previously reported cases did not show any perinatal abnormalities related to fetal hydrops, but a complex vitium cordis (including VSD, ASD, hCMP \& PDA $[3,21]$ ) was diagnosed postnatally in both individuals. We therefore conclude that RIT1 mutations at the same position may result in different phenotypes.

Similar to other Noonan genes, RIT1 is also a member of the RAS superfamily and encodes a protein with GTPase activity. Somatic mutations in RIT1 have been associated with myeloid malignancies and lung adenocarcinoma [22,23]. Thus, we assumed an impact on oncogenesis of RIT1 in gonadoblastoid testicular dysplasia and related gonadoblastoma. In two out of four (of a total of five) gonadoblastoma samples, sequencing analysis revealed mutations in the RIT1 gene. The role of RIT1 in malignancies is unclear so far. Previous investigations 
described heterozygous gain-of-function RIT1 mutations in lung adenocarcinomas and myeloid malignancies, indicating an oncogenic function of RIT1 mutations. In contrast, the two samples with RIT1 mutations (G3 and G4) in our investigation showed two mutations each, consistent with the "two-hit" recessive model of oncogenesis of known tumor suppressor genes. With regard to the function of (the protein encoded by) RIT1, including regulation of cell proliferation and differentiation and furthermore survival in response to oxidative stress, the role of RIT1 as tumor suppressor seems conceivable [24,25]. In line with that, Wakabayashi et al. demonstrated that loss-of function mutations of Rit1 in mice are associated with lymphomagenesis [26].

In conclusion, our data have shown that in terms of tumorigenesis a possible function of RIT1 acting as tumor suppressor gene in humans has to be taken into account. Further investigations of the role of RIT1 in tumorigenesis are needed. Due to the large phenotypic overlap of cases 1 and 2 and the common feature of gonadoblastoid testicular dysplasia, we first investigated RIT1 in case 2, but could not identify any disease causing mutation. Consistent with the clinically suspected diagnosis of Noonan syndrome we subsequently sequenced the PTPN11 gene, revealing a frameshift mutation in exon 10 which led to a premature stop codon. This mutation was previously described neither in cases of Noonan syndrome nor as somatic mutations in malignancies. In contrast, all currently known mutations that cause Noonan Syndrome are missense mutations. Modeling and biochemical data indicate that a gain-of-function of the protein-tyrosine phosphatase SHP2, encoded by PTPN11, is responsible for the phenotype [2]. Besides Noonan syndrome, heterozygous germline mutations in the PTPN11 gene are also described in metachondromatosis and LEOPARD Syndrome [27,28]. Metachondromatosis is a disease characterized by multiple exostosis and enchondromas. LEOPARD is an acronym for the features of this syndrome, specifically: multiple lentigines, electrocardiographic conduction abnormalities, ocular hypertelorism, pulmonary stenosis, abnormal genitalia, growth retardation, and sensorineural deafness. For both genetic diseases putative heterozygous loss-of-function mutations of the PTPN11 gene are described [29,30]. Whereas metachondromatosis shows a completely distinct phenotype, LEOPARD syndrome is often stated to be a subtype of Noonan syndrome, because of the phenotypic overlap. Tartaglia et al. [28] first showed that missense mutations causing LEOPARD syndrome have a dominant negative effect, in contrast to mutations found in cases of Noonan syndrome. That is the reason why it has been suggested to rely for distinction between the two disorders on mutational analysis rather than clinical presentation [29,31]. Even though our case 2, harboring a heterozygous PTPN11 mutation, with a putative loss-of-function, did not show any features typical of LEOPARD syndrome. Symptoms of fetal hydrops associated with polyhydramnios, congenital heart defect, dysmorphic facial features, hydronephrosis and bleeding tendency strongly indicate the diagnosis of Noonan syndrome. The combination of typical Noonan syndrome features with the rare finding of FGTD has not been described in cases of Noonan syndrome to date and may represent a distinct subtype. A review of the literature revealed three cases, including our case 2 that showed FGTD in combination with fetal hydrops and other malformations including coronary heart disease $[8,10]$.

\section{Conclusion}

In the two other cases, however, the underlying genetic mutation has not yet been identified.

We used exome analysis to identify the underlying genetic defect in a fetus with hydrops and gonadoblastoid testicular dysplasia and identified a pathogenic mutation in the RIT1 gene. Variants in RIT1 have been described previously in a rare subtype of Noonan Syndrome. The presentation of FGTD may be an additional feature of a severe form of Noonan syndrome and may even help to establish the diagnosis. Additional analysis of similar cases is needed to provide further evidence.

\section{Acknowledgment}

We would like to thank the parents for consenting to publish these results.

\section{Conflict of interest}

The authors declare no conflict of interest.

\section{References}

1. Roberts AE, Allanson JE, Tartaglia M, Gelb BD (2013) Noonan syndrome Lancet 381: 333-42.

2. Tartaglia M, Gelb BD (2010) Disorders of dysregulated signal traffic through the RAS-MAPK pathway: phenotypic spectrum and molecular mechanisms. Ann N Y Acad Sci 1214: 99-121.

3. Aoki Y, Niihori T, Banjo T, Okamoto N, Mizuno S, et al. (2013) Gain-of-function mutations in RIT1 cause Noonan syndrome, a RAS/MAPK pathway syndrome. Am J Hum Genet 93: 173-80.

4. Bhambhani V, Muenke M (2014) Noonan syndrome. Am Fam Physician 89 $37-43$.

5. Lee KA, Williams B, Roza K, Ferguson H, David K, et al. (2009) PTPN11 analysis for the prenatal diagnosis of Noonan syndrome in fetuses with abnormal ultrasound findings. Clin Genet 7: 190-4.

6. Croonen EA, Nillesen WM, Stuurman KE, Oudesluijs G, Van De Laar IM, et al (2013) Prenatal diagnostic testing of the Noonan syndrome genes in fetuses with abnormal ultrasound findings. Eur J Hum Genet 21: 936-942.

7. Gargano G, Guidotti I, Balestri E, Vagnarelli F, Rosato S, et al. (2014) Hydrops fetalis in a preterm newborn heterozygous for the c. $4 A>G$ SHOC2 mutation. Am J Med Genet A 164A: 1015-1020.

8. Nistal M, Rodriguez JI, Garcia-Fernandez E, Cajaiba MM, Reyes-Mugica M (2007) Fetal gonadoblastoid testicular dysplasia: A focal failure of testicular development. Official Journal of Pediatric and Developmental Pathology. Pediatr Dev Pathol Soc 10: 274-81.

9. Hung NA, Silver MM, Chitayat D, Provias J, Toi A, et al. (1998) Gonadoblastoid testicular dysplasia in Walker-Warburg syndrome. Official Journal of Pediatric and Developmental Pathology. Pediatr Dev Pathol Soc 1: 393-404.

10. Spear GS, Martin CG (1986) Fetal gonadoblastoid testicular dysplasia. Hum Pathol 17: 531-3.

11. Verp MS, Simpson JL (1987) Abnormal sexual differentiation and neoplasia Cancer Genet Cytogenet 25: 191-218.

12. Li H, Durbin R (2010) Fast and accurate long-read alignment with BurrowsWheeler transform. Bioinformatics (Oxford, England) 26: 589-95.

13. Langmead B, Salzberg SL (2012) Fast gapped-read alignment with Bowtie 2 Nature methods 9: $357-9$

14. Sedlazeck FJ, Rescheneder P, Von Haeseler A (2013) Next Gen Map: Fast and accurate read mapping in highly polymorphic genomes. Bioinformatics (Oxford, England) 29: 2790-2791

15. DePristo MA, Banks E, Poplin R, Garimella KV, Maguire JR, et al. (2011) A framework for variation discovery and genotyping using next-generation DNA sequencing data. Nature genetics 43: 491

16. http://picard.sourceforge.net

17. Li H, Handsaker B, Wysoker A, Fennell T, Ruan J, et al. (2009) The sequence alignment/map format and SAM tools. Bioinformatics (Oxford, England) 25: 2078-9.

18. Wang K, Li M, Hakonarson H (2010) ANNOVAR: Functional annotation of genetic variants from high-throughput sequencing data. Nucleic acids res 38: e164. 
Citation: Reischer T, Schmid M, Balendran S, Nistal M, Vodopiutz E, et al. (2016) Fetal Hydrops in Combination with Gonadoblastoid Testicular Dysplasia May Represent a Lethal Type of Noonan Syndrome. Human Genet Embryol 6: 137. doi:10.4172/2161-0436.1000137

19. Popitsch N (2014) CODOC: Efficient access, analysis and compression of depth of coverage signals. Bioinformatics (Oxford, England) 30: 2676-2677.

20. Sanger F, Nicklen S, Coulson AR (1977) DNA sequencing with chain-terminating inhibitors. Proc Natl Acad Sci U S A. 74: 5463-5467.

21. Koenighofer M, Hung CY, McCauley JL, Dallman J, Back EJ, et al. (2015) Mutations in RIT1 cause Noonan syndrome - additional functional evidence and expanding the clinical phenotype. Clin Genet.

22. Berger AH, Imielinski M, Duke F, Wala J, Kaplan N, et al. (2014) Oncogenic RIT1 mutations in lung adenocarcinoma. Oncogene 33: 4418-23.

23. Gómez-Seguí I, Makishima H, Jerez A, Yoshida K, Przychodzen B, et al. (2013) Novel recurrent mutations in the RAS-like GTP-binding gene RIT1 in myeloid malignancies. Leukemia 27: 1943-1946.

24. Shi GX, Cai W, Andres DA (2013) Rit subfamily small GTPases: regulators in neuronal differentiation and survival. Cell Signal 25: 2060-8.

25. Cai W, Rudolph JL, Sengoku T, Andres DA, Rit G (2012) TPase regulates a p38 MAPK-dependent neuronal survival pathway. Neurosci Lett 531: 125-130.

26. Wakabayashi Y, Inoue J, Takahashi Y, Matsuki A, Kosugi-Okano H, et al. (2013)
Homozygous deletions and point mutations of the Rit1/Bcl11b gene in gamma-ray induced mouse thymic lymphomas. Biochem Biophys Res Commun 301: 598-603.

27. Digilio MC, Conti E, Sarkozy A, Mingarelli R, Dottorini T, et al. (2002) Grouping of multiple-lentigines/Leopard and Noonan syndromes on the PTPN11 gene. Am J Hum Genet 71: 389-394.

28. Sobreira NL, Cirulli ET, Avramopoulos D, Wohler E, Oswald GL, et al. (2010) Whole-genome sequencing of a single proband together with linkage analysis identifies a Mendelian disease gene. PLoS Genet 6: e1000991.

29. Kontaridis MI, Swanson KD, David FS, Barford D, Neel BG (2006) PTPN11 (Shp2) mutations in LEOPARD syndrome have dominant negative, not activating, effects. J Biol Chem 281: 6785-6792.

30. Bowen ME, Boyden ED, Holm IA, Campos-Xavier B, Bonafe L, et al. (2011) Loss-of-function mutations in PTPN11 cause metachondromatosis, but not Ollier disease or Maffucci syndrome. PLoS Genet 7: e1002050.

31. Tartaglia M, Martinelli S, Stella L, Bocchinfuso G, Flex E, et al. (2006) Diversity and functional consequences of germline and somatic PTPN11 mutations in human disease. Am J Hum Genet 78: 279-90. 